Preprint n. 1051

October 4, 1994
Dipartimento di Fisica

Università di Roma "La Sapienza"

I.N.F.N. - Sezione di Roma 


\title{
APPROXIMATION METHOD FOR THE PARTITION FUNCTION OF AN ANHARMONIC OSCILLATOR GAS
}

\author{
L. DEFALCO $\left({ }^{2}\right)$, R. MIGNANI $\left({ }^{1}\right)$, R. SCIPIONI $\left({ }^{2}\right)$
}

( ${ }^{1}$ ) Dipartimento di Fisica " E. Amaldi " III Università di Roma, Via C. Segre 2, 00145 Roma ITALY

and

I. N. F. N. - Sezione di Roma I, ¿/o Dipartimento di Fisica, I Università di Roma "La Sapienza", P. le A. Moro 2, 00185 Roma ITALY $\left({ }^{2}\right.$ ) Dipartimento di Fisica " G. Marconi " Università di Roma "La Sapienza", P. le Aldo Moro 2, 00185 Roma ITALY.

We use an approximation procedure to find the partition function of a gas composed by anharmonic oscillators in a region where perturbation theory breaks down. The results are still quite accurate for small values of the coupling constant.

Pacs: $02.60 . \mathrm{Gf} \quad 05.90 .+\mathrm{m} \quad 33.10 . \mathrm{Cs}$

\section{1] Introduction}

There are many problems in quantum mechanics involving anharmonic oscillators; usually, the hamiltonian of the system is put in the form :

$$
\mathrm{H}=\mathrm{H}_{0}+\lambda \mathrm{H}_{1}
$$


where $H_{0}$ and $H_{1}$ are given respectively by:

$$
\begin{aligned}
& H_{0}=\frac{P^{2}}{2}+\frac{\omega^{2}}{2} x^{2} \\
& H_{1}=x^{4}
\end{aligned}
$$

$\lambda$ plays the role of coupling constant while $H_{1}$ is the perturbation. When $\lambda$ is small enough perturbation theory is used to find quantum mechanical results but this procedure breaks down for suitable values of $\lambda[1]$. We want to describe the thermodynamics of a gas composed by anharmonic oscillators exploiting an optimization algorithm, proposed by Burrows, Cohen and Feldman [2]. They introduced a solvable model of Hamiltonian whose spectrum satisfies the relation :

$$
\mathrm{E}_{\mathrm{n}}^{\mathrm{an.osc} .} \geq \mathrm{E}_{\mathrm{n}}^{\bmod \text { el }}(\alpha)
$$

where $\alpha$ is a suitable scaling parameter.

\section{2] The Model}

Let the hamiltonian describing an anharmonic oscillator be written in parametric form as follows :

$$
H(A, B)=\frac{P^{2}}{2}+\frac{A}{2} x^{2}+B x^{4}
$$

and introduce the scale transformation: 


$$
x \rightarrow \frac{x}{\alpha^{1 / 2} B^{1 / 6}}
$$

where $\alpha$ is a suitable scale parameter.

Under trasformation (5) Hamiltonian (4) becomes

$$
H(A, B) \Rightarrow \alpha B^{1 / 3}\left(H_{0}+V\right)=H(A, B ; \alpha)
$$

where

$$
\begin{aligned}
& H_{0}=-\frac{1}{2}\left(\frac{d^{2}}{d x^{2}}-\frac{x^{2}}{2}\right) \\
& V=-\frac{1}{2}\left(1-\frac{A}{\alpha^{2} B^{2 / 3}}\right) x^{2}+\frac{x^{4}}{\alpha^{3}}
\end{aligned}
$$

By a little algebra we get:

$$
\mathrm{V}=\frac{\left[\left(\mathrm{x}^{2}-\gamma\right)^{2}-\gamma^{2}\right]}{\alpha^{3}} \geq-\frac{\gamma^{2}}{\alpha^{3}}=\mathrm{U}
$$

where we introduced:

$$
\gamma=\frac{\alpha^{3}}{4}\left(1-\frac{A}{\alpha^{2} B^{2 / 3}}\right)
$$

Following [2] we obtain a lower bound for the eigenvalues of (4): 


$$
E_{n}(A, B) \geq E_{n}(A, B ; \alpha)=\alpha B^{1 / 3}\left[\left(n+\frac{1}{2}\right)-\frac{\gamma^{2}}{\alpha^{3}}\right]
$$

which can be calculated indipendently for each $n$ using the variational equation :

$$
\frac{d E_{n}(A, B ; \alpha)}{d \alpha}=0
$$

Let us consider now a gas composed by $\mathrm{N}$ anharmonic oscillators whose energies are given by the eigenvalues of (4). The partition function of this quantum gas is given by the usual relation:

$$
\begin{aligned}
& Z=\sum_{\mathbf{n}} e^{-\beta E_{n}(A, B)} \\
& \beta=\frac{1}{K_{B} T} .
\end{aligned}
$$

When $\mathrm{B}$ is great enough we cannot solve the problem exactly but we can use approximation methods; in this way we find some results valid for anharmonic oscillator gas using the energy eigenvalues given by the righthand side of eq. (10), for a suitable value of $\alpha$, which can be found solving (11). Hence the partition function of the scaled hamiltonian (6) can be written as:

$$
Z(\alpha)=\sum_{n} e^{-\beta E_{n}(A, B ; \alpha)}
$$

The relation between (12) and (13) is easily found. Since, by eq. (10), it is: 


$$
E_{n}(A, B) \geq E_{n}(A, B ; \alpha)
$$

we can write :

$$
\sum_{n} e^{-\beta E_{n}(A, B)} \leq \sum_{n} e^{-\beta E_{n}(A, B ; \alpha)}
$$

that means :

$$
\mathrm{Z} \leq \mathrm{Z}(\alpha)
$$

Equation (16) states that (13) is an upper bound to the original partition function which is valid for every positive value of $B$. We argue that, when we get the closest approximation to the energy eigenvalue, we can consider $Z \cong Z\left(\alpha^{*}\right)$ where $\alpha^{*}$ is the value of $\alpha$ which maximizes eqn. (10). After some calculations we get for $\alpha$ the following values :

$$
\begin{array}{ll}
\alpha=\left[4\left(n+\frac{1}{2}\right)\right]^{1 / 3} & A \rightarrow 0 \\
\alpha=\frac{A^{1 / 2}}{B^{1 / 3}} \cos \frac{2 \sqrt{3}}{\omega^{3}} B^{1 / 3}\left(n+\frac{1}{2}\right) & A \rightarrow \infty
\end{array}
$$

Let us recall that $A=\omega^{2}$, ( see eq. (4) ) and therefore the limit $\mathrm{A} \rightarrow 0, \mathrm{~A} \rightarrow \infty$ correspond to low and high frequencies. Then we refer to the two $\alpha$ values $(17 \mathrm{a}, \mathrm{b})$ as $\alpha_{\text {low }}$ and $\alpha_{\text {high }}$ respectively. 


\section{3] Results}

Now we proceed further in the calculation of the partition function. Let's start considering $\alpha_{\text {low }}$. After some algebra we get for $Z$ - function :

$$
Z\left(\alpha_{\text {low }}\right)=\sum_{n} e^{-\beta^{\prime}\left(n+\frac{1}{2}\right)^{4 / 3}}=\sum_{n} e^{-\beta^{\prime} \varepsilon_{n}^{4 / 3}}
$$

where $\beta^{\prime}=1.52 \mathrm{~B}^{1 / 3} \beta$.

Going to the continuum limit we can write :

$$
\begin{aligned}
& Z\left(\alpha_{\text {low }}\right)=\frac{V^{N}}{N ! h^{3 N}}(4 \pi)^{N}\left\{\int_{0}^{\infty} d p p^{2} e^{-\beta^{n} p \frac{8}{3}}\right\}^{N}= \\
& =\frac{(4 \pi)^{N}}{N ! h^{3 N}} V^{N}\left(\frac{3}{8} \beta^{\prime \prime-9 / 8} \Gamma\left(\frac{9}{8}\right)\right)^{N}
\end{aligned}
$$

with $\beta^{\prime \prime}=\frac{\beta^{\prime}}{2^{4 / 3}}=\frac{1.52 B^{1 / 3}}{2^{4 / 3}} \beta$.

Consider now the high - frequency limit. In this case

$$
\alpha=\frac{\omega}{B^{1 / 3}} \cos \frac{2 \sqrt{3}}{\omega^{3}} B^{1 / 3}\left(n+\frac{1}{2}\right)
$$

After some tedious but straightforward calculations we get for the hamiltonian spectrum the expression :

$$
E_{n}\left(\alpha_{\text {high }}\right)=\omega\left(n+\frac{1}{2}\right) \cos \left(\frac{2 \sqrt{3}}{\omega^{3}} B^{1 / 3}\left(n+\frac{1}{2}\right)\right)
$$


which permits, after the passage to the continuum limit, to write the partition function in the form:

$$
Z\left(\alpha_{\text {high }}\right)=\frac{(4 \pi)^{N} V^{N}}{N ! h^{3 N}}\left\{\int_{0}^{\infty} d p \cdot p^{2} e^{-a p^{2} \operatorname{cosb} p^{2}}\right\}^{N}
$$

where $a=\frac{\beta \omega}{2}$ and $b=\frac{\sqrt{3}}{\omega^{3}} B^{1 / 3}$.

Solving the integral at the right hand side of (22) yields:

$$
\begin{aligned}
& Z\left(\alpha_{\text {high }}\right)=\frac{(4 \pi)^{N} V^{N}}{N ! h^{3 N}}\left\{\frac{1}{6 b} \sqrt{\frac{\pi}{a}}\right\}^{N}= \\
& \frac{(4 \pi)^{N} V^{N}}{N ! h^{3 N}}\left\{\frac{\sqrt{2} \omega^{5 / 2}}{6 \sqrt{3} B^{1 / 3}} \beta^{-1 / 2} \sqrt{\pi}\right\}^{N}
\end{aligned}
$$

Eqs. (19), (23) provide the (exact) expression of the partition function of an anharmonic oscillator gas, valid, respectively, for low and high frequencies.

\section{4] Conclusions}

Using the optimization procedure recently proposed by Burrows, Cohen and Feldman, we have been able to calculate the partition function of the anharmonic oscillator gas, in both ranges of high and low values of $\alpha$, where $\alpha$ is an appropriate scaling parameter. The results we have obtained are quite accurate, and promise to have interesting applications, which are currently under investigation. 


\section{REFERENCES}

1] See e. g. S. Bose and D. Tripathy : Fortsch. Phys. 31 (1983) 131.

2] B. L. Burrows, M. Cohen, T. Feldman, J. Math. Phys. 34 (1993) 1. 